\title{
Design principles and performance evaluation of mSCTP-CMT for transport-layer based handover
}

\author{
Łukasz Budzisz, Ramon Ferrús and Ferran Casadevall \\ Radio Communication Group (GCR), \\ Signal Theory and Communication Dept (TSC), \\ Universitat Politècnica de Catalunya (UPC), Spain \\ Email: [lukasz,ferrus,ferranc]@tsc.upc.edu
}

\begin{abstract}
Handling mobility at the transport layer is a promising approach to achieve seamless handover in the context of heterogeneous wireless access networks. In particular, features such as multihoming and dynamic address reconfiguration provided by mobile SCTP (mSCTP) protocol are among the key enablers for handover support at the transport layer. This paper investigates the applicability of Concurrent Multipath Transfer (CMT) to distribute data among two end-to-end paths of a mSCTP association during handover transition process. To that end, the principles of the mSCTP-CMT design are given, emphasizing the consequences of such a sender-introduced reordering and its congestion control implications in a handover scenario. The proposed mSCTP-CMT handover scheme is benchmarked with a pure mSCTP handover scheme. Provided analysis indicates the possible application area of mSCTP-CMT, taking into account not only handover scenario parameters (dwelling time, available bandwidth ratio and round-trip time), but also the most important constraint of such a design: receiver buffer (rbuf) size. Rbuf size proves to be the major limiting factor shrinking significantly possible mSCTP-CMT application scope, yet not excluding definitively the proposed idea.
\end{abstract}

\section{INTRODUCTION}

The Stream Control Transport Protocol (SCTP), defined in RFC 4960 [1] and further referred to as standard SCTP, provides transport-layer multihoming. Multihoming binds multiple source-destination IP addresses for a single association between two SCTP endpoints. These IP addresses are exchanged and verified during the association initiation, and are considered as different paths toward the corresponding peer. Multiple paths are distinguished at each endpoint by their destination addresses. Among all available destinations, one is selected as the primary destination, and the others are used as backup destinations. Multihoming, in the case of IP networks, means multiple IP addresses, and typically (but not necessarily) multiple link-layer interfaces.

Multihoming in SCTP was designed for environments requiring high application availability, such as the delivery of Signaling System No. 7 (SS7) messages. Hence its scope of use, defined within RFC 4960, is only for handling single retransmissions and performing a primary path failover in case of permanent link failure. Any other applications of multihoming, e.g., transport-layer handover or loadsharing are not supported by the standard SCTP specification, and instead should be subject of dedicated protocol extensions. This is the case with transport layer handover that reuses the Dynamic
Address Reconfiguration (DAR) SCTP extension [2], whereas loadsharing is introduced in several different proposals (yet non-standardized by IETF) with the most common scheme called Concurrent Multipath Transfer (CMT) [3]. Standard SCTP enhanced with the DAR extension is referred to as mobile SCTP (mSCTP) [4], [5].

Several studies have already evaluated the performance of the mSCTP in the context of heterogeneous wireless networks. The first analyses investigated the feasibility of mSCTP providing handover support at the transport layer. Authors concluded that mSCTP can supply seamless handover support [6], but still with important shortcomings, even after necessary protocol parameter adjustments [7]. Possibly the main deficiency lies in providing an appropriate handover policy; its design can be approached also in a cross-layer fashion [8]. Budzisz et al. [9] survey mSCTP for transportlayer handovers, and conclude that not only the handover policy but also the improvements introduced to the transition process contribute to override $\mathrm{mSCTP}$ inadequacies. In that sense we propose to evaluate the CMT, as a potential enhancement to the handover schemes based on mSCTP that may smooth the transition process, and moreover, even improve the application's overall throughput. This interesting idea brings back the discussion on SCTP multihoming scope of use, and makes us rethink the original approach once again.

The rest of the paper is organized as follows: Section II provides main assumptions on target scenarios and a description of evaluation approach. Then, Section III discuss the principles of the proposed mSCTP-CMT design. Simulation results and discussion are provided in Section IV, followed by conclusions in Section V.

\section{SCENARIO AND ASSUMPTIONS}

Fig. 1 shows a general handover scenario in heterogeneous wireless networks, where a mobile node ( $\mathrm{MN})$ is moving across one particular radio access network (RAN \#1) coverage area towards the coverage area of a neighboring RAN \#2. The RANs have an overlap area, i.e., an area where both RANs provide coverage. It is assumed that the $\mathrm{MN}$ is capable of handling transmissions on multiple links simultaneously. This assumption is fairly reasonable, as in the near future, many mobile multimedia devices are expected to be equipped with multiple network interfaces, despite the current power 


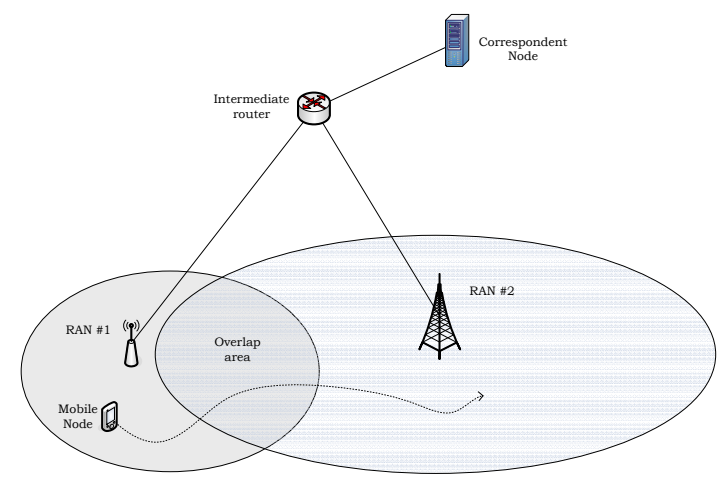

Fig. 1. Proposed CMT scenario.

consumption constraints. Consequently, once the MN enters the overlap area, multiple links are physically available for simultaneous data transmission. This work will examine the possible gain that can be achieved using CMT in the overlap area.

Further, it is also assumed that no shared bottlenecks on the different paths exist, i.e., the radio link of each path is the bottleneck. This is a coherent supposition taking into account the mixed wired-wireless topology of the envisaged scenarios, and as it will be explained later, it is vitally important for the use of CMT. As a consequence, the bottlenecks on each path are independent and a sender can consider a perpath congestion approach, while still preserving overall TCPfriendliness.

Now focusing on the proposed handover scheme, called mSCTP-CMT, this work considers one directional bulk data flow from a correspondent node $(\mathrm{CN})$ to the $\mathrm{MN}$. As presented in Fig. 2, a MN before entering an overlap area is using the mSCTP protocol to transfer data on a single link, configured initially with $\mathrm{IP}_{1}$ address. When the $\mathrm{MN}$ enters the overlap area, the coverage of RAN \#2 is discovered. To get the new link operational, the $\mathrm{MN}$ undergoes the correspondent network registration procedure. Both the network discovery process and registration procedure details are outside the scope of this work. As soon as the network address $\mathrm{IP}_{2}$ in RAN \#2 is operational, the $\mathrm{CN}$ must be informed about the new destination (by means of ASCONF chunk, provided by DAR extension [2]), and has to verify its availability (sending HEARTBEAT (HB) chunk). If the new destination is confirmed, the $\mathrm{IP}_{2}$ address is considered available for normal data transfer. At this point, CMT can be exploited while having two paths available; implementation details are given in Section III. Finally, once the MN leaves the overlap area it is necessary to: (1) quit the CMT mode, (2) handle the retransmissions of the packets that were on flight on the link that just went down, and (3) perform all the necessary congestion adjustments on the current path for the MN which is again being single-homed.

The main goal of this work is to evaluate whether is possible to apply CMT in the presented handover scenario, what gains can be achieved, and in which situations, if any, can the use of CMT degrade service. To this end, the described mSCTP-

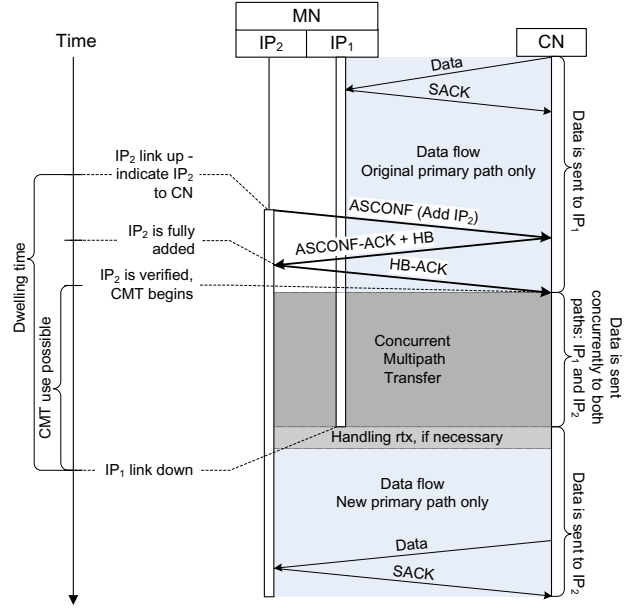

Fig. 2. Handover scheme with mSCTP-CMT.

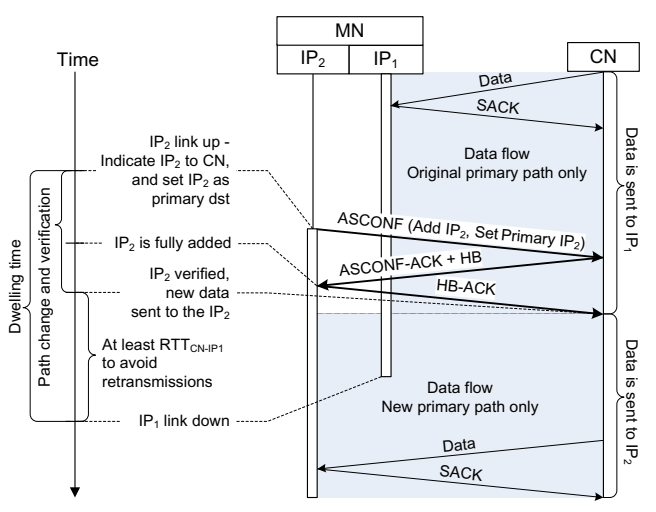

Fig. 3. Handover scheme for mSCTP with handover policy triggering the path change, as soon as the new path is discovered.

CMT handover scheme (Fig. 2) will be compared to the benchmark scheme: a handover based on mSCTP, as illustrated in Fig. 3. As briefly mentioned in Section I, the most important challenge for mSCTP is to provide an appropriate handover policy, i.e., to optimally select the instance when sending the ASCONF chunk with Set Primary parameter should be triggered. Therefore, it may be considered beneficial for some handover policies to bundle Set Primary with Add/Delete IP Address parameter within one ASCONF chunk. To give the reader more insight on this issue, two ideal schemes reflecting range of possible adjustments to the envisaged scenario are provided: (1) the best case, with policy resulting in MN staying in the better quality RAN (in terms of bandwidth, propagation delay or both) as long as possible, and (2) the worst case keeping the $\mathrm{MN}$ in the poorer quality RAN for the maximum time.

Lastly, two important parameters to evaluate mSCTP-CMT 
performance are named:

1) dwelling time $\left(t_{\text {dwell }}\right)$, defined as the effective time a $\mathrm{MN}$ remains in the overlap area. Dwelling time is affected by the speed of the MN, as well as its movement pattern, and therefore may be crucial for using CMT. Depending on the scenario considered $t_{d w e l l}$ can vary from few seconds for fast MNs going across the overlap area to tenths of seconds for slow MNs moving within the overlap area.

2) bandwidth ratio ( $\left.b w_{\text {ratio }}\right)$, defined as a ratio of the bandwidth available on the newly added path in RAN \#2 (that may be used for CMT), and the original RAN \#1 path's bandwidth available before entering the overlap area. bw $w_{\text {ratio }}$ parameter reflects the asymmetry of a heterogeneous scenario in function of considered RANs.

\section{DESIGN CONSIDERATIONS FOR A MSCTP-CMT SCHEME}

To introduce CMT into a mSCTP-based handover scheme, a few important aspects must be considered. First, standard SCTP's congestion control algorithms must be updated to take into account the problems of sending data over multiple paths using the single sequence number space. The consequences of sender-introduced reordering are described in Section III-A. Second, in wireless scenarios, the fixed Internet's basic assumption that losses are generally due to congestion is invalid. Wireless links are more prone to packet corruption than to congestion problems. Therefore, it is reasonable to expect failures more often in wireless scenarios, and to reduce their impact by applying the CMT's Potentially Failed (CMT-PF) solution proposed in [10] that will be described in Section III-B. The CMT scheme under test described in Section IV will comprise both modifications.

\section{A. Congestion control modifications}

Accomplishing CMT for SCTP implies a new sender architecture, where each path (not necessarily interface) must have a separate buffer to guarantee path independence. This modification preserves TCP-friendliness under the assumption that the bottleneck is not shared by the path, which is the case in the analyzed scenario (a mixed wired-wireless topology with single hop wireless part). Of course, such a multi-buffer sender structure has its implications on congestion control, and therefore several changes must be considered. All algorithms cited here and incorporated to our analysis, were proposed by Iyengar et al. [3]:

1) congestion control is handled per-path, not per association, so it is possible to have different congestion window (cwnd) evolution schemes, as functions of the conditions on each path. Thus, Selective Acknowledgments (SACKs) updating the Cumulative Transmission Sequence Number ACK Point (CumTSN) received inorder per path and out-of-order per association should increase the cwnd on that path. To track the earliest outstanding TSN per destination, a sender cwnd growth algorithm (cwnd update for CMT - CUC) has been proposed.

2) fast retransmission needs slight modification as reordering introduced on the sender side can provoke unnecessary spurious fast retransmissions with cwnd implications. Elimination of spurious fast retransmissions is handled by the Split Fast Retransmit (SFR) algorithm.

3) receiver should not send immediate SACKs on the arrival of duplicate packets as networks may be vulnerable for the increased ACK traffic. Therefore an algorithm Delayed ACK for CMT (DAC) was applied.

4) appropriate retransmission policy for handling retransmissions. Out of five different policies proposed by Iyengar in [3], the best results for bulk transfer were achieved by the loss rate-based policies. One of them, RtxCwnd policy (path with the highest cwnd handles the retransmission), will be used in presented experiments.

Besides previous considerations, CMT can provoke the following problems that must be taken into account when considering mSCTP-CMT scheme:

1) Receiver buffer (rbuf) blocking (receiver buffer is filled with out-of-order data) caused by complete or short-term failures. This problem is partially mitigated by the CMTPF solution described in Section III-B.

2) An ambiguity at the sender for the SACKs with the same CumTSN that acknowledge various Gap ACK blocks: first more Gap ACK blocks are acked on the faster path followed then by a packet with fewer Gap ACK blocks received on the slower path. This can lead to an unnecessary retransmission in case the difference between paths bandwidths is big.

3) Incorrect round-trip time (RTT) estimate on a slower path that comes from the ambiguity of the SACK received on the faster path that also acknowledges the packet marked for a RTT estimation on the slower path.

\section{B. CMT-PF extension}

To reduce the rbuf problem in CMT the Potentially Failed (PF) solution was proposed in [10]. The path that has experienced a failure (single timeout) is marked as a potentially failed and no more data transmission is allowed on that path. Instead, a HB packet is sent every RTO to probe this path, and either the path gets back to an active state in case of one successful HB packet transmission, or the path is considered inactive after the Path.Maximum.Retransmissions (PMR) threshold is exceeded (with $\mathrm{PMR}+1$ consecutive failures). The PF state prevents the PMR parameter settings from degrading the throughput performance during failure scenarios, as the exponential backoff mechanism clocks only the HB packets.

\section{PERformance evaluation}

To study the feasibility of mSCTP-CMT for transportlayer handover, a set of simulation experiments in ns-2 (ver. 2.32) [11] was conducted, adjusting an existing CMT-PF implementation to work with heterogeneous wireless environments. Proposed scenario under test (Fig. 1) considers the 
TABLE I

SIMULATION PARAMETERS

\begin{tabular}{|c|c|}
\hline PARAMETER NAME & VALUE / RANGE \\
\hline Wired part (each path) & $\begin{array}{r}\text { bandwidth: } 100 \mathrm{Mbps} \\
\text { propagation delay: } 5-20-45-90 \mathrm{~ms}\end{array}$ \\
\hline Fast RAN & $\begin{array}{r}\text { bandwidth: }\left(\text { b } w_{\text {ratio }} \times 384\right) \mathrm{kbps} \\
\text { propagation delay: } 15 \mathrm{~ms}\end{array}$ \\
\hline Slow RAN & $\begin{array}{r}\text { bandwidth: } 384 \mathrm{kbps} \\
\text { propagation delay: } 80 \mathrm{~ms}\end{array}$ \\
\hline bw $w_{\text {ratio }}$ & $1-14$ \\
\hline$t_{d w e l l}$ & $2-40 \mathrm{~s}$ \\
\hline rbuf size & $\begin{array}{r}16-256 \mathrm{kB} \\
\text { (ideal buffer up to } 2 \mathrm{MB} \text { ) }\end{array}$ \\
\hline RTOMin & $50 \mathrm{~ms}$ \\
\hline PMR & $\begin{array}{r}\text { mSCTP: 1 } \\
\text { mSCTP-CMT: } 5 \\
\end{array}$ \\
\hline Retransmission policies & $\begin{array}{l}\text { mSCTP FastRtx: Same path } \\
\text { mSCTP TimeoutRtx: Alternate path } \\
\text { CMTRtx: path with largest cwnd }\end{array}$ \\
\hline MTU size / Data payload & $1500 / 1468$ Bytes \\
\hline File size & $8 \mathrm{MB}$ \\
\hline
\end{tabular}

following mobility pattern: (1) first the MN moves within the coverage area of RAN \#1, (2) after $t_{1}$ from the transmission start the $\mathrm{MN}$ enters the overlap area where there is a possibility of applying CMT scheme for the time $t_{d w e l l}$, and finally (3) MN moves to the RAN \#2 coverage area, where again only one path is available for data transmission. Two types of transitions are analyzed: (1) from fast to slow RAN, and (2) from slow to fast RAN. Additionally, four different values of propagation delay are taken into account to evaluate the mSCTP-CMT performance in various RTT conditions. Summary of the most important simulation parameters is presented in Table I. Note that proposed RANs settings could correspond to WLAN for fast RAN, and UMTS for slow RAN, respectively.

First, a performance comparison in terms of TSN-time evolution diagram is presented for two mentioned types of transition: fast-to-slow RAN (Fig. 4a) and slow-to-fast RAN (Fig. 4b) for both handover schemes described in Section II, namely: (1) mSCTP-based solution (both best and worst case), and (2) the mSCTP-CMT scheme (which includes CMT-PF, but for short is referred to as mSCTP-CMT). As can be seen the transition type is not a factor having much influence in the presented scenario. In both cases the 40s overlap area (2-42 s in the time scale) is the zone of special interest, witnessing different slope values for presented SCTP flavors. Any possible CMT gain over pure MSCTP-based handover schemes will occur here, if strict constraints on rbuf size are met. The range for possible mSCTP-CMT performance gain is significant, from being much worse than the best mSCTP policy (nearly as bad as the worst one) in presence of rbuf blocking, to gaining over the best mSCTP policy for an appropriate rbuf size adjustment. Still in any of the considered cases mSCTP-CMT did not perform any worse than the worst mSCTP policy. In an effort to draw more exact application area for mSCTP-CMT, all important factors such as, $t_{d w e l l}, b w_{\text {ratio }}$ and rbuf size will be analyzed in presence of various RTT

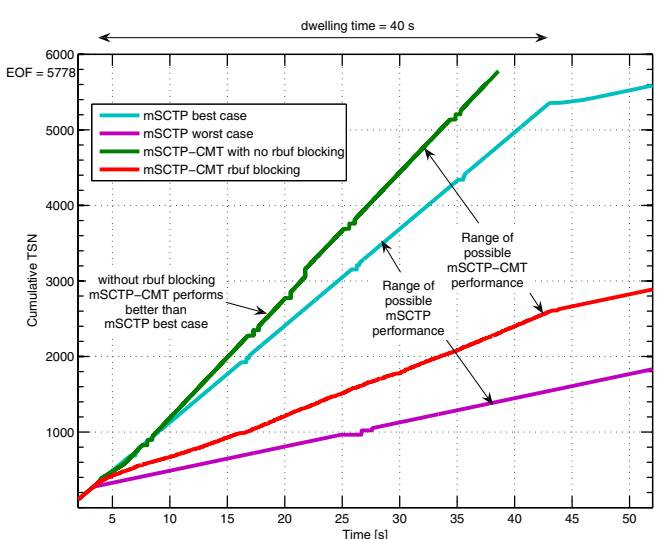

(a)

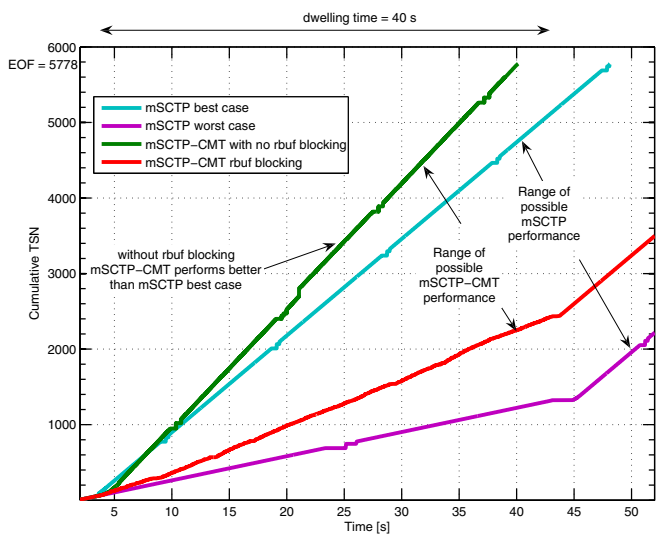

(b)

Fig. 4. Performance comparison of all handover schemes for b $w_{\text {ratio }}=4$, and $t_{\text {dwell }}=40$ s and for: (a) fast to slow RAN; and, (b) slow to fast RAN transition.

values. Since the mobility pattern does not seem the factor influencing much the mSCTP-CMT performance only one mobility pattern (fast-to-slow RAN) was selected to conduct further experiments.

To better understand the rbuf blocking problem, in Fig. 5, the following metrics are provided: the smallest rbuf size that guarantees mSCTP-CMT outperforms MSCTP in terms of overall file transfer, for worst and best policy accordingly, as well as the smallest rbuf size without the rbuf blocking problem at all. The result is clear, not much asymmetry between two paths is allowed. Provided that $256 \mathrm{kB}$ limitation on rbuf memory at MN seems reasonable nowadays, only a $b w_{\text {ratio }}$ less than two makes the mSCTP-CMT application feasible, if the design concern is not having rbuf blocking at all. Less conservatively, if improvement over the mSCTP best case is the sole design goal, mSCTP-CMT scope of use extends to $b w_{\text {ratio }}$ of three, or even four in function of considered RTT, value that would correspond, for instance, to a handover from WLAN to UMTS. Beyond this limit, the difference between both links makes the application of mSCTP-CMT pointless. This is hold true for both short and long $t_{d w e l l}$, therefore only the result for long $t_{d w e l l}$ are provided in Fig. 5. 


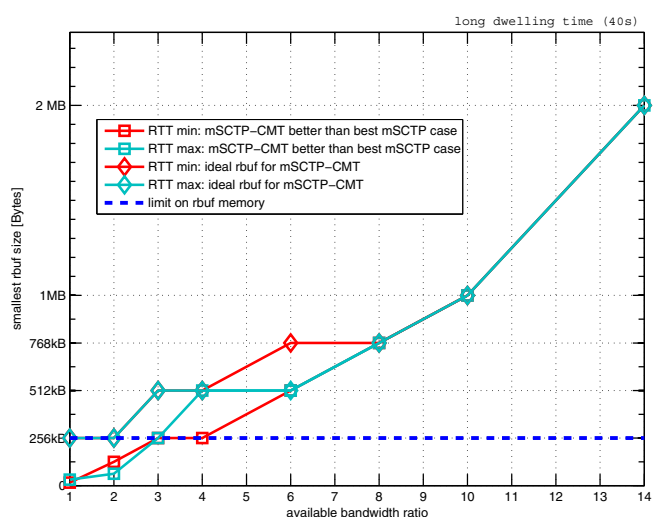

Fig. 5. Rbuf size constraints for long $t_{d w e l l}(40 \mathrm{~s})$.

As seen in dwelling time graph (Fig. 6), users with short $t_{d w e l l}$ would have no significant gain from using mSCTPCMT when compared to mSCTP-based schemes in any of presented cases. In contrast, having long $t_{d w e l l}$ can effectively benefit from mSCTP-CMT. However, again the impact of rbuf blocking can be witnessed; within tested scenario mSCTPCMT was not capable of outperforming best mSCTP case for a rbuf not exceeding $256 \mathrm{kB}$ for b $w_{\text {ratio }}$ equal to 4 . For $b w_{\text {ratio }}=2$ already $128 \mathrm{kB}$ buffer guaranteed better performance than mSCTP for all investigated values of RTT. As there was not much difference in the performance of mSCTP-CMT for four considered RTT cases (according to the Table I) only the boundaries results were marked in Fig. 5-6.

\section{CONCLUSION}

In this paper we have analyzed the applicability of CMT (or strictly speaking CMT-PF) to the mSCTP-based transportlayer handover scenarios, as having the potential of smoothing the handover process between two paths. Provided that mSCTP itself lacks handover policies, CMT can be seen as an added value to such mSCTP scheme. An initial evaluation proves strong influence of the receiver buffer blocking on any scenario where $\mathrm{mSCTP-CMT}$ is used, resulting in firm limitation of possible application area in terms of receiver buffer size. The next important factor, scenario asymmetry, measured here as the available bandwidth ratio, puts also strong constraints on design of such system. Still, as initial results demonstrate, all of these requirements can be met in some heterogeneous scenarios.

\section{ACKNOWLEDGMENT}

This work was supported in part by CYCIT (Spanish National Science Council) under grant TEC2006-09109.

\section{REFERENCES}

[1] R. Stewart, "RFC 4960, Stream Control Transmission Protocol (SCTP)," September 2007. [Online]. Available: http://www.ietf.org/rfc/rfc4960.txt

[2] R. Stewart, Q. Xie, M. Tuexen, S. Maruyama, and M. Kozuka, "RFC 5061, Stream Control Transmission Protocol (SCTP) Dynamic Address Reconfiguration," September 2007. [Online]. Available: http://www.ietf.org/rfc/rfc5061.txt

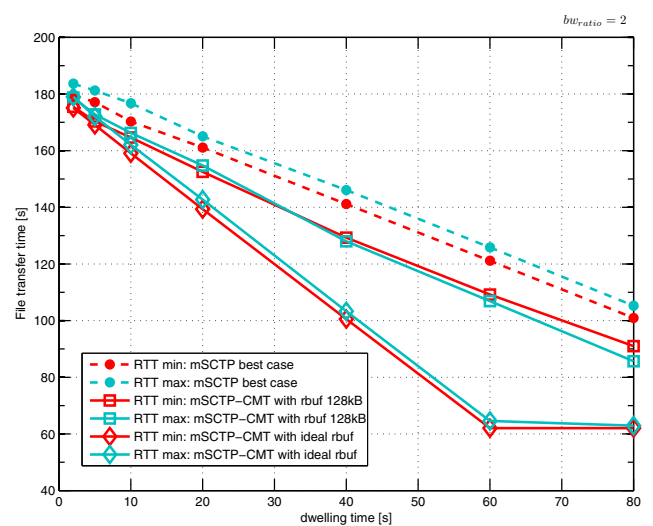

(a)

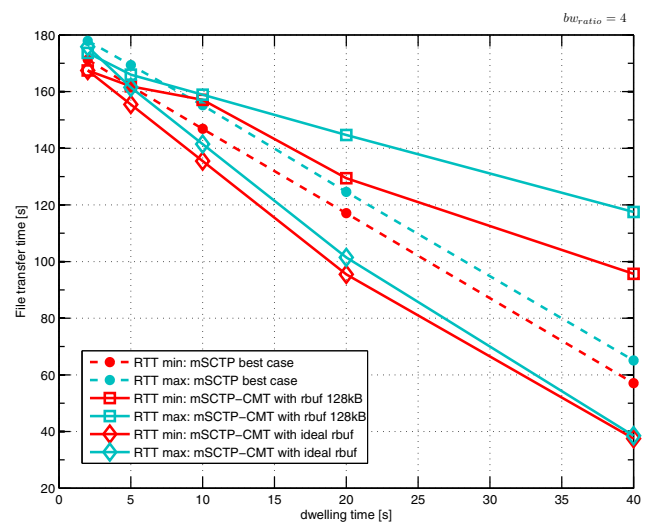

(b)

Fig. 6. Comparison for different RTT values in function of dwelling time

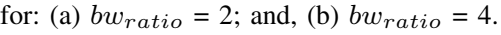

[3] J. Iyengar, P. Amer, and R. Stewart, "Concurrent multipath transfer using sctp multihoming over independent end-to-end paths," IEEE/ACM Transactions on Networking, vol. 14, no. 5, pp. 951-964, October 2006.

[4] M. Riegel and M. Tuexen, "Mobile SCTP, IETF draft," November 2007. [Online]. Available: http://tools.ietf.org/id/draft-riegel-tuexenmobile-sctp-09.txt, work in progress

[5] S. J. Koh and Q. Xie, "Mobile SCTP (mSCTP) for IP handover support, IETF draft," October 2005. [Online]. Available: http://tools.ietf.org/id/draft-sjkoh-msctp-01.txt, draft expired

[6] L. Ma, F. Yu, V. Leung, and T. Randhawa, "A new method to support UMTS/WLAN vertical handover using SCTP," IEEE Wireless Communications, vol. 11, no. 4, pp. 44-51, August 2004.

[7] Ł. Budzisz, R. Ferrús, and F. Casadevall, "SCTP multihoming performance in dynamically changing channels with the influence of link-layer retransmissions," in The 64th IEEE Vehicular Technology Conference (VTC 2006-Fall), September 2006, pp. 2624-2628.

[8] M. Chang, M. Lee, and S. J. Koh, "Transport layer mobility support utilizing link signal strength information," IEICE Transactions on Communications, vol. E87-B, no. 9, pp. 2548-2556, September 2004.

[9] Ł. Budzisz, R. Ferrús, A. Brunstrom, R. Grinnemo, K.-J. Fracchia, G. Galante, and F. Casadevall, "Towards transport-layer mobility: Evolution of sctp multihoming," Computer Communications, vol. 31, no. 5, pp. 980-998, March 2008.

[10] P. Natarajan, J. Iyengar, P. Amer, and R. Stewart, "Concurrent multipath transfer using transport layer multihoming: Performance under network failures," in IEEE Military Communications Conference (MILCOM 2006), October 2006.

[11] "The network simulator ns-2." [Online]. Available: http://www.isi.edu/nsnam/ns/ 